\title{
Effect of Emotional Labor on Somatization among Dental Hygienists in an Area
}

\author{
Mi-Jeong $\mathrm{Kim}^{\dagger}$, Cha-Young Lim, and Ju-Lee Son \\ Department of Dental Hygiene, Vision College of Jeonju, Jeonju 55069, Korea
}

\begin{abstract}
Background: We investigated somatization symptoms experienced by dental hygienists due to stress from emotional labor. Our aim was to provide basic research data that could be useful in the development of efficient stress management schemes for this occupational group.

Methods: We analyzed data collected from 208 dental hygienists working in Jeollabuk-do Province, Korea. To measure the level and intensity of emotional labor among research participants, we used the Korean Emotional Labor Questionnaire. We used the Somatization Symptom Checklist-90-Revised (SCL-90-R) to measure the level of somatization symptoms among participants.

Results: On analyzing the level of emotional labor and somatization symptoms according to general characteristics, participants aged $23 \sim 25$ years showed high scores for stress due to emotional labor $(p<0.05)$. Working 5 days per week, subjective health status, and organizational support and protection systems were found to correlate with the level of stress due to emotional labor $(p<0.05)$. In the analysis of correlations between emotional labor and somatization symptoms, scoring high across all domains of emotional labor was associated with scoring high for somatization symptoms in the subdomains of emotional labor. When emotional labor and demographical variables were used as independent variables, having higher scores for emotional labor and having poor subjective health status were found to be associated with having higher levels of somatization symptoms $(\mathrm{p}<0.05)$.

Conclusion: Our results showed that working at night and organizational support and protection systems were correlated with emotional labor and somatization symptoms. Measures must be taken at the organizational level to reduce emotional labor and somatization symptoms.
\end{abstract}

Key Words: Dental hygienist, Emotional labor, Somatic symptom

\section{Introduction}

The improvement of medical technology and quality of life in the era of the Fourth Industrial Revolution calls for qualitative improvement of medical services for patients who visit the hospital ${ }^{1)}$. In addition, optimized convergence of health and medical care requires not only medical treatment but also professional care by people with a pleasing personality and good expertise, which are considered interpersonal services needed to ensure satisfaction among patients. As a result, dental clinics emphasize the role of the dental hygienist, who provides comprehensive dental services to patients with their expertise and skills ${ }^{2}$. Dental hygienists are responsible for helping maintain oral health and prevent periodontal diseases and dental caries through the application of fluoride, tooth fillings, provisional fillings, temporary attachment and removal, tooth patterning, fitting and removal of orthodontic wires, scaling, and so on. However, dental hygienists also play a role in increasing dental service satisfaction. Dental hygienists must often adjust their emotions according to their patients' feelings when receiving medical services rather than their own feelings, in order to increase patients' satisfaction with the medical services ${ }^{3)}$. It has been reported that dental hygienists experience a lot of stress while working with increases in emotional labor practices that emphasize these medical services ${ }^{4}$. Emotional labor is a feeling of being stressed owing to expressing the emotions desired 
by patients and the organization and concealing the emotions of the worker ${ }^{5}$. Emotional labor is a factor generated in performing a job, and an increase in emotional labor leads to increased stress and lower job efficiency ${ }^{6}$. According to a survey on emotional labor of the Korea Employment Information Service, the level of emotional labor among dental hygienists ranked 14th among 730 occupational workers in Korea. Dental hygienists were also ranked third for emotional labor among occupations that deal with patients or appealing to people $^{7)}$. Dental hygienists' emotional labor is increased due to regulating his/her feelings according to the patient's feelings, regardless of the actual emotions they may be experiencing; this causes job stress and this stress results in somatic symptoms such as headache, stomachache, indigestion, and fatigue. If not treated properly at an early stage, somatization can lead to chronic conditions that are not easy to treat and can also cause poorer performance of dental tasks ${ }^{8-10)}$. In addition, it has been reported that stress owing to emotional labor is a main cause of health deterioration among dental hygienists as well as in their job capability and productivity at the dental $\operatorname{clinic}^{11}$. Therefore, it is necessary to prevent and manage stress caused by emotional labor and somatization symptoms arising while dental hygienists are at work. A previous study on the emotional labor of dental hygienists has reported that turnover intention and work organization affect emotional labor in clinical dental hygienists ${ }^{12)}$, and the level of dental hygienists' emotional labor is influenced by their personality type ${ }^{13)}$. Although many studies have examined the effects of emotional labor on job stress ${ }^{14)}$, research on the effects of emotional labor with respect to job stress and somatization among dental hygienists is lacking.

We conducted this study to provide basic data for the development of efficient management schemes, aimed at improving the mental and physical health of dental hygienists, by analyzing symptoms of stress owing to dental hygienists' emotional labor.

\section{Materials and Methods}

\section{Research participants and methods}

\section{1) Research participants}

The participants in this study were dental hygienists working in Jeollabuk-do Province, selected using a convenience sampling method. The total number of participants was 204; we used the $G^{*}$ Power 3.1 program to calculate the minimum sample size after setting a significance level of 0.05 , an effect size of 0.15 , and a power of 0.95 . A total of 220 participants were selected for the study, considering sample bias and a dropout rate of $10 \%$, to reduce sampling error. We finally analyzed data for a total of 208 of 220 respondents who agreed to complete the questionnaire after receiving a verbal description of the purpose of the study. We excluded the data of 12 respondents that were inappropriate due to incomplete answers. The study period was from January 28, 2019 to February 28, 2019. This study was approved by the Wonkwang University Bioethics Committee (WKIRB-201901-SB-006).

\section{2) Research tools}

To measure the level and intensity of emotional labor among research participants, we used the Korean Emotional Labor Questionnaire ${ }^{15)}$. Emotional labor was assessed using 24 questions in 5 domains: 5 questions regarding "requirements and regulations of emotion control", 3 questions addressing "overload and conflict related to customer service", 6 items querying "incongruity and impairment of emotional labor", 3 questions on "observation and monitoring of organization", and 7 questions addressing "organizational support and protection system". A reliability test was conducted to determine the internal consistency of the emotional labor questionnaire, resulting in a Cronbach's $\alpha$ value of 0.869 . Cronbach's $\alpha$ values for the five domains of emotional labor were calculated as follows: 0.745 for "requirements and regulations of emotion control", 0.721 for "overload and conflict related to customer service", 0.868 for "emotional incongruity and impairment", 0.823 for "observation and monitoring of organization", and 
0.792 for "organizational support and protection system".

Somatization is a physical discomfort or symptom and a tendency to appeal despite no convincing pathological causes, as well as recognizing the symptom as a physical disease and seeking medical help. We used the Symptom Checklist-90-Revised (SCL-90-R) to measure the level of somatization symptoms among participants ${ }^{16)}$. This survey tool uses a somatization scale including 12 questions. A reliability test showed a Cronbach's $\alpha$ value of 0.865 .

The emotional labor and somatization symptom survey tool includes a 5-point Likert scale and all comprised of negative items, which means that a higher score indicates worse emotional and somatization symptoms.

The general characteristics of participants such as age, highest educational level, work experience, job position, working hours, working 5 days per week, working at night, monthly average income level, sleeping time, and subjective health status were investigated.

\section{3) Data analysis}

The independent t-test and one-way ANOVA were used to identify differences in emotional labor and somatization symptoms according to the general characteristics of participants, and the Scheffe test was conducted as a post test. The correlation between emotional labor and somatization symptoms was analyzed using Pearson's correlation coefficient. In addition, multiple regression analysis was performed to analyze factors affecting somatization symptoms. The data collected in this study were analyzed using PASW Statistics ver. 18.0 (IBM Corp., Armonk, NY, USA). The significance level $(\alpha)$ used in the statistical test was 0.05 .

\section{Results}

\section{Differences between emotional labor and somatization symptoms according to general characteristics}

Table 1 shows differences in emotional labor and somatization symptoms according to the general characteristics of the dental hygienists included in our study. The overall level of emotional labor was statistically significant for the characteristics of age, working 5 days per week, and subjective health status $(\mathrm{p}<$ $0.05)$. The age group $23 \sim 25$ years showed the highest score for emotional labor (80.06) and the age group 36 40 years had the lowest score (66.60). In addition, the emotional labor score was high (80.92) among respondents who did not have a 5-day work week. Regarding subjective health status, the survey score (82.74) indicated poor subjective health status.

Scores for the emotional labor subdomains according to the general characteristics of dental hygienists were statistically significant $(p<0.05)$ for age, working at night, and subjective health status in the domain "requirements and regulations of emotion control." The age group $31 \sim 35$ years had the highest score (20.29). The highest score for working at night (19.6) was for the period from $8 \mathrm{pm}$ to $8: 30 \mathrm{pm}$. The highest score for subjective health status was 20.34 .

In the domain "overload and conflict related to customer service", a significant difference $(\mathrm{p}<0.05)$ was found for job position, average monthly income, and subjective health status. With a higher job position, the average monthly income was higher but subjective health status was also poorer. In the case of "emotional incongruity and impairment", age and subjective health status were significant $(\mathrm{p}<0.05)$, with the highest score (21.26) among dental hygienists aged 23 25 years and scores indicating poorer subjective health status. In the domain “organizational support and protection system", age, working 5 days per week, working at night, and subjective health status were statistically significant $(\mathrm{p}<0.05)$. The age group $41 \sim 50$ years showed the highest score at 22.71 ; scores were 22.35 among respondents who did not have a 5-day work week and 22.29 among those who did not work at night. In addition, scores indicated poorer subjective health status among respondents.

Somatization symptoms according to general participant characteristics were significant for subjective health status $(p<0.05)$. When dental hygienists perceived that their subjective health status was poor, the somatization symptom score was high, at 52.03 . 


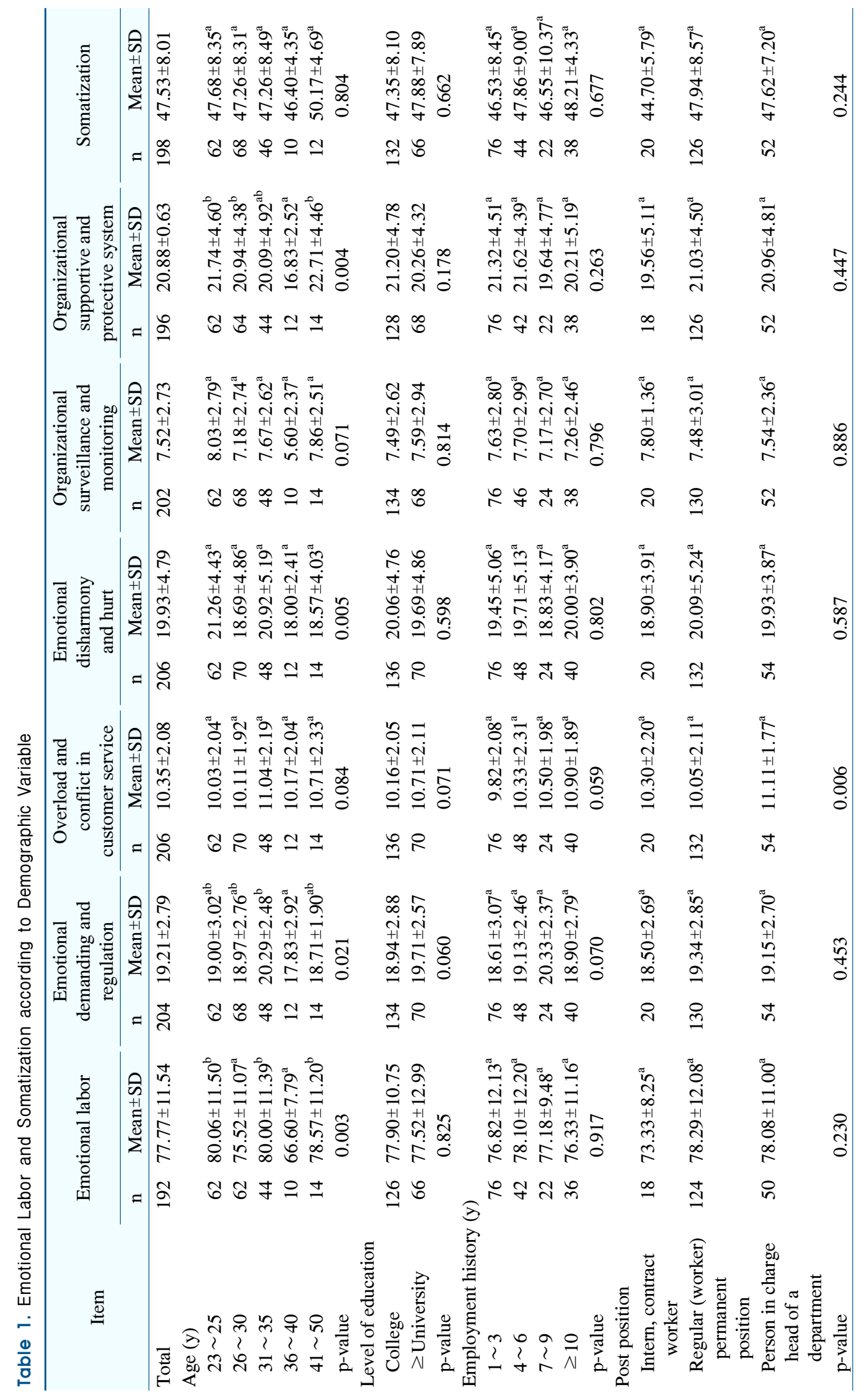




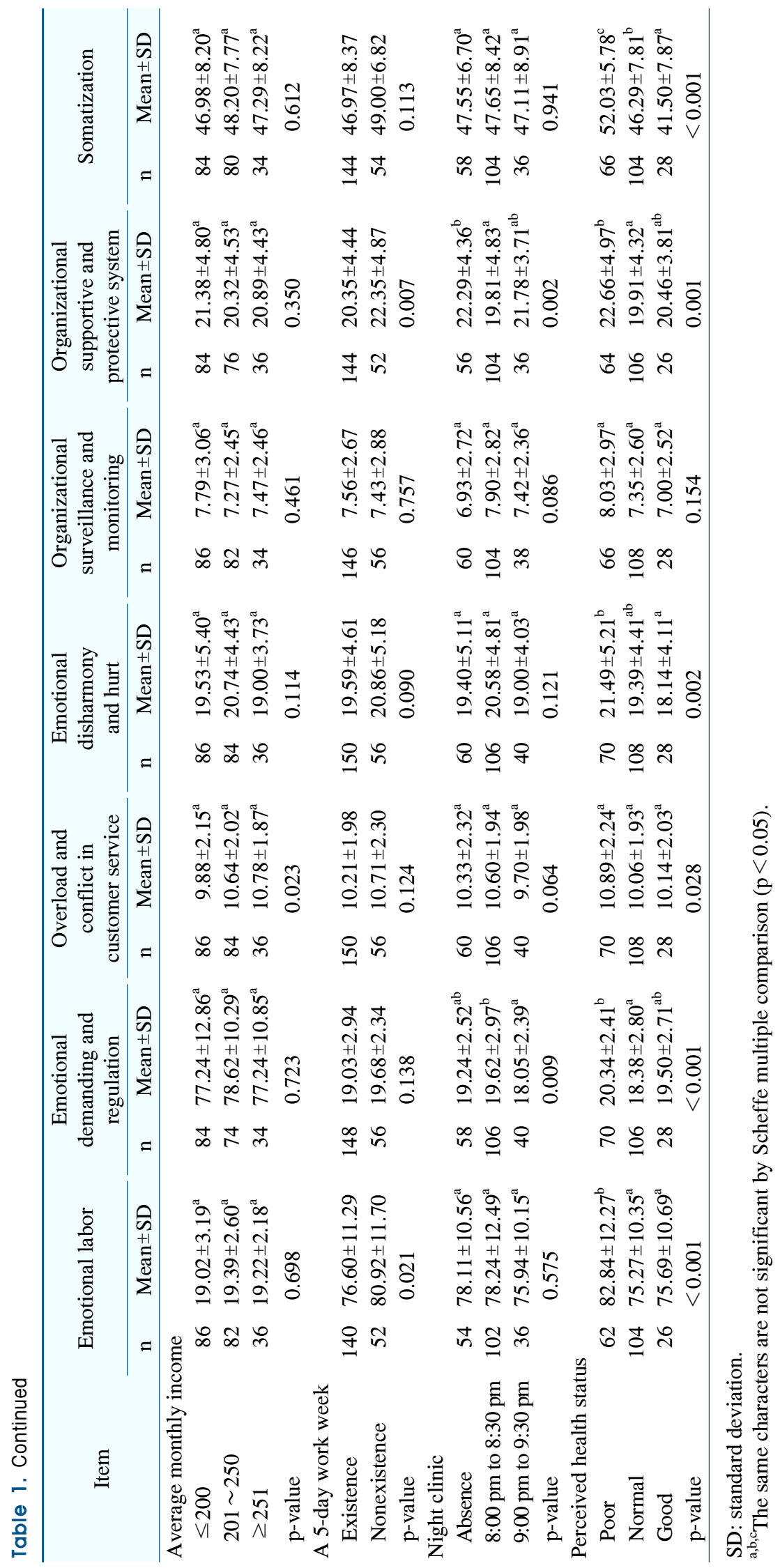




\section{Correlation between emotional labor and somatization symptoms}

The analysis results for correlation of somatization symptoms are shown in Table 2 . There was a significant correlation with somatization for both overall emotional labor score and emotional labor subdomains $(\mathrm{p}<0.001)$. With higher overall emotional labor score and higher scores in the emotional labor subdomains, somatization symptom scores were also higher.

\section{Analysis of factors affecting emotional labor and sociodemographic characteristics in somatization symptoms}

Table 3 shows analysis of the factors affecting emotional labor and sociodemographic characteristics with respect to somatization symptoms. In Model 1, where emotional labor was used as an independent variable, higher emotional labor score $(\beta=0.562)$ was associated with higher somatization symptoms.

In Model 2, in which demographic variables were used as independent variables, not having a 5-day work week ( $\beta=0.168)$ and poorer subjective health status $(\beta=-0.531)$ were correlated with higher somatization symptom score.
In Model 3, where emotional labor and sociodemographic variables were used as independent variables, higher emotional labor score $(\beta=0.458)$ and poorer subjective health status $(\beta=-0.388)$ were associated with higher somatization symptoms, with power of the model $42.4 \%$.

\section{Discussion}

The Fourth Industrial Revolution has brought many changes to the health and medical fields. In the medical field, segmentation of new technologies and medical services suitable for the era of convergence of these fields is increasing. As a consequence of these social changes, the combination of technical and emotional aspects of dental hygienists' work has led to the development of a new medical service area. With these complex changes, emotional labor of dental hygienists continues to increase in line with improvement in the quality of medical services. Emotional labor of dental hygienists is associated with stress, which is often expressed as somatization symptoms. Therefore, we conducted this study to provide basic data on the development of programs suitable for the

Table 2. Correlation between Emotional Labor and Somatization

\begin{tabular}{lcccccc}
\hline & $\begin{array}{c}\text { Total } \\
\text { emotional labor }\end{array}$ & $\begin{array}{c}\text { Emotional } \\
\text { demanding } \\
\text { and regulation }\end{array}$ & $\begin{array}{c}\text { Overload } \\
\text { and conflict in } \\
\text { customer service }\end{array}$ & $\begin{array}{c}\text { Emotional } \\
\text { disharmony } \\
\text { and hurt }\end{array}$ & $\begin{array}{c}\text { Organizational } \\
\text { surveillance and } \\
\text { monitoring }\end{array}$ & $\begin{array}{c}\text { Organizational } \\
\text { supportive and } \\
\text { protective system }\end{array}$ \\
\hline Somatization & $0.554^{* *}$ & $0.198^{* *}$ & $0.366^{* *}$ & $0.592^{* *}$ & $0.319^{* *}$ & $0.314^{* *}$ \\
\hline
\end{tabular}

$* * \mathrm{p}<0.001$ by Pearson's correlation analysis.

Table 3. Effects of Emotional Labor, Demographic Variable on Somatization

\begin{tabular}{|c|c|c|c|c|c|c|}
\hline & \multicolumn{2}{|c|}{ Model 1} & \multicolumn{2}{|c|}{ Model 2} & \multicolumn{2}{|c|}{ Model 3} \\
\hline & $\beta$ & $\mathrm{p}$ & $\beta$ & $\mathrm{p}$ & $\beta$ & $\mathrm{p}$ \\
\hline Total emotional labor & 0.562 & $<0.001$ & & & 0.458 & $<0.001$ \\
\hline Age (y) & & & -0.075 & 0.550 & 0.068 & 0.545 \\
\hline Level of education & & & -0.071 & 0.338 & -0.063 & 0.339 \\
\hline Employment history & & & -0.012 & 0.938 & 0.043 & 0.755 \\
\hline Post position & & & 0.097 & 0.326 & 0.001 & 0.989 \\
\hline Average monthly income & & & 0.164 & 0.128 & 0.032 & 0.748 \\
\hline A 5-day work week & & & 0.168 & 0.022 & 0.091 & 0.165 \\
\hline Night clinic & & & 0.051 & 0.500 & 0.078 & 0.261 \\
\hline \multirow[t]{2}{*}{ Perceived health status } & & & -0.531 & $<0.001$ & -0.388 & $<0.001$ \\
\hline & \multicolumn{2}{|c|}{$\begin{array}{c}\mathrm{R}^{2}=0.316 \\
\text { Adjusted } \mathrm{R}^{2}=0.312\end{array}$} & \multicolumn{2}{|c|}{$\begin{array}{c}\mathrm{R}^{2}=0.282 \\
\text { Adjusted } \mathrm{R}^{2}=0.248\end{array}$} & \multicolumn{2}{|c|}{$\begin{array}{c}\mathrm{R}^{2}=0.454 \\
\text { Adjusted } \mathrm{R}^{2}=0.424\end{array}$} \\
\hline
\end{tabular}


convergence era, to improve somatization symptoms among dental hygienists and mediate the stress of emotional labor by analyzing the effects of this stress on somatization symptoms.

First, the results of emotional labor intensity and somatization symptoms according to general characteristics of the participants revealed a high emotional labor stress score of 80.06 in the age group 23 25 years. This finding is consistent with previous studies by Won and $\mathrm{Oh}^{17)}$ and Lee and $\mathrm{Oh}^{18)}$ who reported high social and psychological stress among participants with younger age and shorter work experience. We investigated whether there is support or a protection system for dental hygienists when problems arise while engaged in emotional labor during medical service provision. The score for the age group $41 \sim$ 50 years was high at 22.71 , which was similar to the results of Choi et al. ${ }^{19)}$. It is believed that the leadership of dentists and positive support from senior and fellow workers are very important to mitigate various stressors and emotional labor among dental hygienists. The results of this study indicate that with older age, participants feel less protected by the internal system, whereas among those who are younger and have fewer working years, the clinical adaptation training and attention is more supportive. It seems that dental hygienists are not receiving the benefit of internal protection systems because older hygienists have greater responsibility and independence. Thus, we consider that a positive atmosphere and support are needed for mutual cooperation within the dental organization. We found that implementing a 5-day work week was associated with subjective health status as well as organizational support and protection systems. The score for somatization symptoms was high when respondents' subjective health condition was perceived as poor. Scores for emotional labor and somatization symptoms were also high when a 5-day work week was not implemented. Given that dental hygienists must perform their work while standing all day, working more than 52 hours per week can lead to increased frequency of emotional labor, which is directly associated with somatization symptoms. According to the correlation between emotional labor and somatization symptoms, the results of analysis using demographic variables as the independent variables showed that somatization symptoms were higher when respondents' subjective health condition was perceived as poor and they did not work 5 days per week. In addition, subjective health status was found to be poor when working at night. As a result, we consider that working late at night without implementing a 5-day work week could worsen somatization symptoms among dental hygienists and deteriorate their health condition, which may interfere with their ability to perform effective dental hygiene practice. According to the research results of Jeong et al. $^{20)}$, a 5-day work week is reported to improve work satisfaction. Therefore, it is believed that dental clinics can improve the performance of dental hygienists by making changes in the working environment such as implementing a 5-day work week system and making adjustments such that medical treatment is not provided very late at night. In addition, dental hygienists with greater responsibilities, such as department heads and team leaders, had high stress scores (11.11) in the "overload and conflicts related to customer service" domain; this is seen as a result of the interaction with patients owing to direct, one-on-one patient communication, patient care, and overall counseling with higher job positions. As many non-insurance dental services have a relatively high treatment cost, dental hygienists with greater job responsibilities must often consult with patients. The dental hygienist must engage in emotional labor during this process, and relatively high competition with nearby dental clinics requires them to provide friendly and patient medical services. This increases the stress from emotional labor among dental hygienists, who must suppress their emotions and perform emotional labor as required by the clinic ${ }^{21)}$. As we have found in this study, the correlation between emotional labor and somatization symptoms showed that the higher the overall emotional labor score, the higher the somatization symptom score in emotional labor subdomains. Moreover, the effect of emotional labor and demographic variables on somatization symptoms showed that higher emotional labor score and worse subjective health status were associated with higher somatization symptoms. Lee and $\mathrm{Chi}^{9}{ }^{9}, \mathrm{Kim}^{10)}$, and Yoon and $\mathrm{Kim}^{11)}$ also reported that 
somatization symptoms were more likely to lead to more frequent poor performance among dental hygienists; this is consistent with our result that a higher score for emotional labor was correlated with a higher somatization symptom score.

As a result of the above research, we suggest that appropriate measures such as support and protection systems of the organization as well as worker welfare programs should be established, to understand and reduce the emotional labor experienced by dental hygienists.

The limitation of this study is that the results cannot be generalized to all of Korea as the data were collected from dental hygienists in only some areas of the country. Further systematic studies are needed with diversification of sampling, to examine the correlation with variables of dental hygienists' emotional labor, stress, and somatization symptoms. However, our study findings can be used as basic data for identifying the symptoms of somatization and the causes of stress related to emotional labor among dental hygienists and the core workforce in dental health services in the era of converging health and medical services. We consider that reducing stress caused by emotional labor and alleviating somatization symptoms among occupational groups such as dental hygienists, by developing programs to reduce this stress, proper workforce management, and improving organizational support and protective systems, as well as worker welfare in dental clinics, could improve the quality of medical services and enhance work efficiency among dental hygienists.

\section{Notes}

\section{Conflict of interest}

No potential conflict of interest relevant to this article was reported.

\section{Ethical approval}

This study was approved by the Wonkwang University Bioethics Committee (WKIRB-201901-SB-006).

\section{ORCID}

Mi-Jeong Kim, https://orcid.org/0000-0002-5309-1853

\section{Cha-Young Lim, https://orcid.org/0000-0001-9345-7349}

Ju-Lee Son, https://orcid.org/0000-0003-4611-8891

\section{Acknowledgements}

This research was supported by funds of Vision College of Jeonju.

\section{References}

1. Oh YJ, Choi YH: Effects of emotional labor, job stress and burnout on somatization in nurses: in convergence era. J Digit Converg 13: 415-424, 2015. https://doi.org/10.14400/JDC.2015.13.10.415

2. Kang $\mathrm{OH}$ : The determinants of intent to leave of dental hygienists at dental clinics. J Dent Hyg Sci 6: 107-111, 2006.

3. Lee CS, Park SY, Han HM, et al.: Influential job-related characteristics for the job satisfaction and turnover possibility of dental hygienists. J Dent Hyg Sci 12: 666-674, 2012.

4. Choi HJ, Bang HJ, Chung EY, Seo YJ: Factors that affect the level of emotional labor in dental hygienists. J Dent Hyg Sci 14: 295-301, 2014.

5. Hochschild AR: Emotion work, feeling rules, and social structure. Am J Soc 85: 551-575, 1979.

6. Kim MJ, Lim CY: Relationships between experiencing verbal violence and the emotional responses and coping behaviors of dental hygienists. J Dent Hyg Sci 17: 209-217, 2017. https://doi.org/10.17135/jdhs.2017.17.3.209

7. Retrieved October 14, 2015, from http://www.keis.or.kr/user/ bbs/main/137/775/bbsDataView/27976.do.

8. Park MM, Han SJ: Relations of job satisfaction with emotional labor, job stress, and personal resources in home healthcare nurses. J Korean Acad Community Health Nurs 24: 51-61, 2013. https://doi.org/10.12799/jkachn.2013.24.1.51

9. Lee JY, Chi MW: Relation among emotional labors's job stress, role conflict, ego-resilience and job turnover. J Korea Content Assoc 12: 191-200, 2012. https://doi.org/10.5392/JKCA.2012.12.06.191

10. Kim IS: The role of self-efficacy and social support in the relationship between emotional labor and burn out, turn over intention among hospital nurses. J Korean Acad Nurs Admin 15: 515-526, 2009.

11. Yoon SU, Kim JS: Relations between emotional labor and 
job stress among some dental hygienists. J Korean Soc Dent Hyg 11: 178-188, 2011.

12. Lee MJ, Kim YN, Lee SY: A study on factors affecting emotional labor of clinical dental hygienists. J Dent Hyg Sci 15: 113-118, 2015.

https://doi.org/10.17135/jdhs.2015.15.2.113

13. Yoon SU, Nam IS: Relations between emotional labor and sleep health according to the personality types of dental hygienists. J Dent Hyg Sci 11: 243-249, 2011.

14. Hwang DH, Eom S: The convergence influence of emotional labor of clinical dental hygienist working on job stress: focused on mediating effects of self esteem. J Korea Converg Soc 7: 67-74, 2016.

15. Jang SJ, Kang HT, Kim SY, et al.: A study on the application of the Korean type of emotional labor and violence (study of 2013). Korea Occupational Safety \& Health Agency, Ulsan, pp.1-128, 2014.

16. Kim KI, Kim JH, Won HT: Korean mental of symptom checklist-90-revision. Jung Ang Juk Sung Publisher, Seoul, pp.8-10, 1984

17. Won YS, Oh HN: The effects of job stress and psychosocial stress among dental hygienists. J Dent Hyg Sci 15: 679-687, 2015. https://doi.org/10.17135/jdhs.2015.15.5.679

18. Lee KM, Oh HJ: The effect of a five-day working week on employee's job attitudes. Korean Bus Educ Rev 203-222, 2008.

19. Choi YK, Kim KY, Kim MS, Moon BA, Kim KH: The relationship between emotional labor status and depression in dental hygienists. J Korea Acad-Ind Cooper Soc 18: 635-643, 2017. https://doi.org/10.5762/KAIS.2017.18.7.635

20. Jeong SB, Choi EM, Choi JS: The effects of emotional labor on burnout, turnover intention, and job satisfaction among clinical dental hygienists. J Korean Acad Oral Health 38: 50-58, 2014. https://doi.org/10.11149/jkaoh.2014.38.1.50

21. Kang HK, Kwun HS: A study on job satisfaction according to clinical dental hygienists' career in Busan. J Korean Acad Dent Hyg Educ 9: 507-521, 2009. 